\title{
Enhancement in QoS for Hybrid Networks Using IEEE 802.11e HCCA with Extended AODV Routing Protocol
}

\author{
Shalini Singh ${ }^{1}$, Rajeev Tripathi ${ }^{2}$ \\ ${ }^{1}$ Department of Electronics and Communication Engineering, Graphic Era University, Dehradun, India \\ ${ }^{2}$ Department of Electronics and Communication Engineering, MN National Institute of Technology, \\ Allahabad, India \\ Email: shalini111.raj@gmail.com
}

Received 20 May 2015; accepted 26 June 2015; published 30 June 2015

Copyright (C) 2015 by authors and Scientific Research Publishing Inc.

This work is licensed under the Creative Commons Attribution International License (CC BY). http://creativecommons.org/licenses/by/4.0/

(c) (i) Open Access

\begin{abstract}
The mobile ad hoc network (MANET) with infrastructure networks (hybrid networks) has several practical uses. The utility of hybrid network is increased in real time applications by providing some suitable quality of service. The quality thresholds are imposed on parameters like end-toend delay (EED), jitter, packet delivery ratio (PDR) and throughput. This paper utilizes the extended ad hoc on-demand distance vector (AODV) routing protocol for communication between ad hoc network and fixed wired network. This paper also uses the IEEE 802.11e medium access control (MAC) function HCF Controlled Channel Access (HCCA) to support quality of service (QoS) in hybrid network. In this paper two simulation scenarios are analyzed for hybrid networks. The nodes in wireless ad hoc networks are mobile in one scenario and static in the other scenario. Both simulation scenarios are used to compare the performance of extended AODV with HCCA (IEEE 802.11e) and without HCCA (IEEE802.11) for real time voice over IP (VoIP) traffic. The extensive set of simulations results show that the performance of extended AODV with HCCA (IEEE 802.11e) improves QoS in hybrid network and it is unaffected whether the nodes in wireless ad hoc networks are mobile or static.
\end{abstract}

\section{Keywords}

MANET, HCCA, Extended AODV, Hybrid Network, Quality of Service

\footnotetext{
"Corresponding author.
}

How to cite this paper: Singh, S. and Tripathi, R. (2015) Enhancement in QoS for Hybrid Networks Using IEEE 802.11e HCCA with Extended AODV Routing Protocol. Int. J. Communications, Network and System Sciences, 8, 236-248. 


\section{Introduction}

Interconnection of mobile ad hoc networks (MANETs) to IP networks extends the coverage of mobile network, increases the network capacity, and finally, expands the communication and the application range of ad hoc networks. Nowadays this issue is acquiring major importance due to the possibility of extending the Internet beyond its traditional scope to remote inaccessible areas making web services available anytime and anywhere. To obtain connectivity between infrastructure network and ad hoc wireless network (hybrid network), it is necessary to introduce an Internet Gateway that provides the link to external hosts. Thus, a gateway acts as a bridge between a MANET and the infrastructure network. All communication between the two network domains must pass through gateway. This paper utilizes the modified version of ad hoc on-demand distance vector (AODV) routing protocol which is known as extended AODV. Extended AODV routing protocol is able to route packets not only within a mobile ad hoc network, but also to infrastructure network [1]. Although, the Internet Engineering Task Force (IETF) has proposed several routing protocols for pure MANETs, which were designed for communication within an autonomous MANET. Those routing protocols are unsuitable for MANET with infrastructure network or hybrid network.

The hybrid network's utility is enhanced by the support quality of service (QoS) requirements [2]. There are still more considerable research efforts to be done in this area. In order to support the QoS requirements to hybrid networks in terms of guaranteed bandwidth and bounded delay and jitter, the IEEE 802.11 Working Group for wireless local area network (WLAN) Standards has produced the release of the IEEE 802.11e amendment. The IEEE 802.11e amendment introduces traffic and service differentiation at the MAC layer so that it can deliver multimedia streams with the respect of their QoS and real-time requirements, in addition to regular best effort traffic [3]. The 802.11e introduces the hybrid coordination function (HCF) and defines two channel access mechanisms. The first one is enhanced channel distributed access (EDCA) based on contention-based distributed coordination function (DCF) channel access by introducing traffic prioritization [4]. The other is a controlled channel access referred to as HCF controlled channel access (HCCA). The controlled channel access is a polling-based scheme enhanced from point coordination function (PCF) of 802.11. The HCCA mechanism [5] uses a QoS-aware centralized coordinator, called hybrid coordinator (HC), and operates under some rules that are different from the point coordinator (PC) of the PCF. HCCA enhances the optimal Point Coordination Function (PCF) polling scheme with a parameterized traffic classification [6] [7].

This paper analyses the performance of extended AODV with 802.11e HCCA to support MANET with infrastructure networks. To achieve this aim, two simulation scenarios are considered in this paper: one is that when the nodes are mobile and the other is when nodes are static in wireless ad hoc network domain. The simulation results prove that extended AODV with IEEE802.11e HCCA is beneficial for both the scenarios in real time application.

The rest of the paper is organized as follows: Section 2 gives an overview of most relevant research work. Section 3 includes brief description of the extended AODV routing protocol [8]. In Section 3.1, we illustrate the IEEE802.11e MAC protocol. In Section 4, simulation scenarios with their parameters values are explained. Section 4.1 provides the performance metrics used for proposed simulation scenarios and detailed description of simulation results. Section 5 presents conclusion of the paper.

\section{Related Work}

There have been a lot of researches on providing QoS in MANET with infrastructure networks. The MANET with infrastructure network is supported by an Internet gateway that provides the necessary configuration parameters to the mobile nodes. Among the work that is based on the family of IEEE 802.11 standards and focus on distributed solutions and provide only service differentiation and not QoS guarantees. The work related to QoS guarantees through resource reservation in MANET with infrastructure networks is presented below.

A. Hamidian [1] utilizes the Extended AODV routing protocol to route packets, not only within a MANET but also between a wireless MANET and the wired Internet. The gateway nodes are used to communicate between the ad hoc wireless and the wired network. The reactive, proactive and hybrid gateway discovery method are implemented and compared. Therefore, the results presented are valid for the specific scenario. The author concludes that none of the gateway discovery methods is the best one for every possible scenario. Ali Hamidian et al. [2] proposed scheme, which is called EDCA with Resource Reservation (EDCA/RR), operates in a fully distributed manner. This scheme is compatible with IEEE 802.11, and provides both prioritized and paramete- 
rized QoS. The EDCA/RR is extended to handle reservation collisions. The simulation results reported that the proposed scheme can handle multiple reservations as well as uninformed stations that lie outside the transmission range of both the transmitter and the receiver while providing QoS guarantees. It has been also compare EDCA/RR with EDCA. The comparison results concludes that as the traffic in the network increases, EDCA/RR succeeds in providing the required service to QoS-demanding applications whereas EDCA fails in this task. It has been also proven that when the medium is lossy, EDCA/RR gives better service to real-time traffic and provides contention to non-real-time traffic. Ali Hamidian et al. [4] presented a distributed MAC scheme based on 802.11e for providing QoS guarantees in WLANs operating in ad hoc mode. One advantage with this solution is that it regulates the medium access with a distributed admission control algorithm. Moreover, there is a resource reservation mechanism allowing the stations wishing to send traffic with strict QoS requirements to reserve TXOPs. The proposed scheme has been compared to 802.11e's contention-free medium access method, the EDCA, which cannot provide any strict QoS guarantees. The simulation results verified that proposed scheme performs better than the EDCA except when the traffic load is very light.

Hua Liang et al. [7] analyzed quality of service (Qos) scheduling mechanism of IEEE802.11e and the simple scheduling algorithm (Simple Scheduler). It explore the Simple Scheduler deficiencies exist in supporting variable bit-rate service (VBR). Hence, the author proposed an improved algorithm which adapts to the real time VBR service. The algorithm can dynamically adjust the service interval (SI) and the transmission opportunity (TXOP) of per flow. The proposed algorithm is implementing and compared with existing HCCA. Simulation results show that proposed mechanism not only effectively improved the delay and transmission efficiency but also meets the need of real time QoS.

Shalini et al. [8] utilizes extended Ad hoc On Demand Distance Vector (AODV) for interconnection of MANET with Internet. An approach is presented to reduce the search cost; such as route discovery delay (RDD) and routing overhead (RO) based on modification in TTL value for network wide search. Moreover, this paper not only simulates and compares the existing and proposed approach for optimization in search cost but also derive search strategies (i.e. sequence of TTL values). By the use of dynamic programming approach, with which search strategies can be derived to minimize the search cost. The mathematical derivation for the class of TTL based search method also justifies the obtained simulation results for MANET with infrastructure network. This paper also concludes that there is no effect on the performance of extended AODV, whether the source mobile node uses the home domain gateway or foreign domain gateway to send the data packets to the wired domain.

Anna Lina Ruscelli et al. [9] presented novel local node scheduler, named Overboost. This Overboost is suitable for improving performance of HCCA schedulers of IEEE 802.11e networks by exploiting the EDCA function. This Overboost, uses HCCA to negotiate a minimum bandwidth and deals with traffic streams that require more bandwidth than the negotiated one by redirecting the excess bandwidth to the EDCA function. The analytical evaluation shows that it efficiently uses the wireless medium in presence of different types of traffic, and reduces the length of the HCCA queue. These results has been validated through simulations showing that Overboost improves the QoS support provided by the HCCA schedulers when variable bit rate traffic streams are transmitted. In particular it increases the algorithm efficiency expressed in terms of the null rate, throughput, access delay and queue length.

As the real-time applications in wireless networks, need some schemes to provide more suitable service for them [10]. The referenced scheduling scheme provided in IEEE 802.11e standard, does not perform well on traffic which is not strictly CBR. Therefore, it is required to design a more flexible scheme to dynamically adjust the estimation of TXOP allocated to QSTAs with different characteristics of applications. Hence, this paper presents a data rate estimation algorithm for the scheduler of the IEEE 802.11e HCCA and a simple queue-lengthbased weighted compensating time allocation. The aim of the proposed algorithm is to provide bounded delay for both VBR and CBR traffic. The simulation results show that the proposed scheme is good for both VBR and CBR traffic and is stable for various load conditions comparing with the referenced scheduling scheme and the contention-based access method. Therefore, the proposed scheme provides efficient way to estimate fluctuating data rate and provide delay, packet loss rate, and throughput guarantee in error free wireless environment. If the channel condition is varying with time, the proposed scheme needs to combine other techniques to lower the BER, like link adaptation. Finally, the polling-based scheme is much more suitable to operate under interference-free conditions, while contention- based scheme can still operate normally when there are other WLANs within the range.

Ali Hamidian et al. [11] described the functionalities of IEEE 802.11e EDCA and introduced EDCA/RR, 
which is a medium access scheme based on EDCA. The EDCA/RR offers Traffic Stream's (TSs) with QoS requirements and offers the opportunity to reserve Transmission Opportunity's (TXOPs) for deterministic and contention-free medium access. In other words, EDCA/RR extends EDCA by allowing for reservation of periodic TXOPs. The scheme uses a distributed approach for admission control, scheduling and reservation, making its implementation rather easy. Moreover, it is based on existing standards.

\section{Extended AODV or AODV+}

When a mobile node from ad hoc wireless network domain wish to communicate with infrastructure domain node (or Internet), mobile node searches its routing table for route towards destination node in infrastructure domain. If a route is found, the mobile node starts sending packets. Otherwise, the mobile nodestarts a route discovery process if it does not find a route to the infrastructure station (node) in its routing table. The route discovery process is started by broadcasting a Route Request (RREQ). If a route is found, the intermediate node would normally send a RREP back to the originator of the RREQ. But in that case, the source node would think that the destination is a mobile node that can be reached via the intermediate node. It is important that the source knows that the destination node is located at infrastructure network and not a mobile node from ad hoc wireless network, because these are sometimes processed differently. The extended AODV routing protocol has been solved this problem by preventing the intermediate node to send a RREP back to the originator of the RREQ if the destination node is in the infrastructure network. Instead, the intermediate node updates its routing table and rebroadcasts the received RREQ message. To determine whether the destination node is located in infrastructure network not an intermediate node in MANET. The source mobile node has to consult its routing table. If the next hop address of the destination is a default route (see Table 1), the destination node is situated in infrastructure network. Otherwise, the destination is a mobile node or a gateway. Since neither the infrastructure node nor the mobile nodes in the MANET can reply to the RREQ, it is rebroadcasted until its TTL value reaches zero. When the timer of the RREQ expires, a new RREQ message is broadcasted with a larger TTL value. However, since the fixed node cannot receive the RREQ message (no matter how large the TTL value is) the source will never receive the RREP message it is waiting for. This problem has been solved by letting the source mobile node assume that the destination node is in infrastructure network if a network wide search has been done without receiving any corresponding RREP. In that case, the source must find a route to a gateway (if it does not have one already) and send its data packets towards the gateway, which will forward them towards the fixed node. A considerable amount of route discovery delay will occur with expanding ring search if the destination node is located in infrastructure domain. Modifying the parameters involved in the expanding ring search technique (such as TTL_START and TTL_THRESHOLD) can decrease the route discovery delay if the destination node is located in infrastructure domain. However, this modification can also result in increased routing overhead if the destination is a mobile station. Assuming the destination node is in infrastructure network, increasing TTL_START equal to the TTL_THRESHOLD value would result in less number of broadcasted RREQs and, consequently, delay in discovery process is considerably reduced. This modification in TTL value is known as network-wide search, which is beneficial to find the route from source mobile node in ad hoc wireless network to destination node in infrastructure network. The routing table should be updated after a network-wide search without receiving any corresponding RREP. Once the source has determined that the destination is fixed node in infrastructure network (Internet), it has to create a route entry for the fixed node in its routing table. The first entry of Table 1 tells the node that the destination is a fixed node since the next hop is specified by the default route. The second entry specifies which gateway the node has chosen for its Internet connection. The last entry gives information about the next hop towards the gateway. Table 1 shows the routing table of a mobile node after creating a route entry for a fixed node.

The challenge is how to setup the routing table of an intermediate mobile node (IMN) chosen to forward data

Table 1. Table routing table of mobile node.

\begin{tabular}{cc}
\hline Destination Address & Next Hop Address \\
\hline Fixed node & Default \\
Default & Gateway \\
Gateway & IMN \\
\hline
\end{tabular}


packets towards the gateway. Since the forward route entries are created for the gateway (the source of the RREP_I) and not for the fixed node, which is the final destination of the data packets. An IMN will not find any valid route for the fixed node when it receives data packets from the source mobile node. Therefore, it would normally drop the data packets because it does not know how to forward them. In extended AODV, if IMN does not find a valid route to the destination and if the destination is a fixed node, it creates a (or updates the) route entry for the fixed node in its routing table and forwards the data packets towards the gateway.

\section{IEEE 802.11e HCCA}

The traditional IEEE 802. 11MAC standard cannot provide real-time services and did not address the QoS issues sufficiently [12]. When the station is polled in IEEE 802.11 MAC, it can only send a frame once a time, and with no priority. Therefore, the 802.11 working group formed task group E, which is 802.11e [13], an important enhancement of 802.11. IEEE 802.11e standard which introduces a new QoS mechanism: HCF (Hybrid Coordination Function), which contains two modes, namely EDCA (Enhanced Distributed Channel Access) [11] and HCCA (HCF Controlled Channel Access) [9]. EDCA can only be used in CP (Contention Period), while HCCA can be used both in CP and CFP (Contention-Free Period). EDCA is basically an improved mechanism for DCF, and HCCA is basically an improved mechanism for PCF [14]. EDCA cannot provide any QoS guarantees; only service differentiation. On the other hand, HCCA can provide QoS guarantees through resource reservation but it is a centralized and more complex scheme, which is useful in infrastructure networks only [10]. HCCA transfer protocol defines a time parameter which is called transmission opportunity (TXOP) [15], which refers to a time duration during which a Quality of Service Station (QSTA) is allowed to transmit a burst of data frames. These bounded time intervals were introduced to solve the problem with unknown transmission times of polled stations in PCF. HCCA TXOP is calculated according to TSPEC (Traffic Specification) sent by each QSTA, then use the CF-Poll frame to transmit to each QSTA. Transmission different traffic classes called traffic streams (TSs) are introduced in HCCA. TSPEC (Traffic Specification) is added information element in IEEE 802.11e standard. The QoS request frame includes a Traffic Specification (TSPEC) element (see Table 2) that brings the information to notify the requirements of the traffic stream (TS). This simple scheduler uses the mandatory set of TSPEC parameters to generate a schedule. A TSPEC describes the QoS characteristics of a traffic stream (TS) by specifying parameters such as Mean Data Rate, Service Interval (SI), Delay Bound, Nominal SDU size etc. Each TS can either be unidirectional or bi-directional (or both of them), corresponds to a specific service level identified by the values of the Traffic Specification (TSPEC) protocol parameters. In order to control the delay, the maximum value of a TXOP is bounded by a value called TXOP Limit, which is determined by the Quality of Service Access Point (QAP). A QSTA can transmit multiple frames within its TXOP allocation. A QSTA never allowed exceeding the TXOP $\mathrm{P}_{\text {limit }}$ imposed by the QAP, including interframe spaces and acknowledgements. This new feature also tends to provide time-based fairness between QSTAs. The Service Interval (SI), which is the time interval between two successive polls of the node, and the transmission opportunity (TXOP) which is the node transmission duration, based on the mean application data rates of its TSs.

Admission control is required to limit the amount of existing data traffic in the network and prevent the network to become overloaded. However, the task of implementing an admission control and scheduling scheme is not defined in the 802.11e standard. The standard only presents guidelines for the design of a simple scheduler and admission control unit (ACU). Therefore, some of researches are proposed enhancements to the reference design in IEEE 802.11e. In 802.11e, the scheduler and ACU use the mandatory set of TSPEC parameters to decide on admission and generate a schedule:

- $\rho$ : Mean Data Rate (from the negotiated TSPEC);

- L: Nominal MSDU Size (from the negotiated TSPEC);

- SImax: Maximum Service Interval or D: Delay Bound;

- R: Physical Transmission Rate (equal to the Minimum PHY Rate negotiated in the TSPEC or the observed PHY Rate);

Table 2. TSPEC element fields.

\begin{tabular}{ccccc}
\hline TS Info & Nominal MSDU Size & Max. MSDU Size & Min. Service Interval Max. Service Interval & Inactivity Interval \\
\hline Mean Data Rate & Peak Data Rate & Max. Burst Size & Delay Bound & Min. Physical Rate
\end{tabular}


- M: Maximum Allowable Size of MSDU (i.e., 2304 bytes);

- O: Overheads in time units (including interframe spaces, ACK frames and CF Poll frames).

The admission control and scheduling procedure can be described according to the following four steps:

1. Calculate SI. First the scheduler calculates the minimum $m$ of all SImax for all admitted streams. Then SI equals a value lower than $\mathrm{m}$ and a submultiple of the beacon interval.

2. Calculate the number of MSDUs that arrive at the mean data rate during SI. For TS i, where L and $\rho$ are given by the application, the number of arrived MSDUs during SI equals.

$$
N_{i}=\left\lceil\frac{\operatorname{SIx} \rho_{i}}{L_{i}}\right\rceil
$$

3. Calculate the TXOP duration. The scheduler calculates the TXOP duration as the maximum of (a) the time to transmit $N_{i}$ frames at $R_{i}$ plus overhead and (b) the time to transmit one maximum size MSDU at $R_{i}$ plus overhead. This way, the scheduler ensures that the station can transmit at least one maximum-sized MSDU during a TXOP. Thus, the TXOP duration for TS $i$ equals

$$
\operatorname{TXOP}_{i}=\max \left(\frac{N_{i} L_{i}}{R_{i}}+O, \frac{M}{R_{i}}+O\right)
$$

4. Admit or reject the TS. Assuming that there are $k$ admitted streams, a new stream $(k+1)$ can be admitted if it satisfies the following inequality:

$$
\frac{T X O P_{k+1}}{S I}+\sum_{i=1}^{k} \frac{T X O P_{i}}{S I} \leq \frac{T-T_{C P}}{T}
$$

\section{Simulation Setup}

Two simulation scenarios created to evaluate the performance of extended AODV with HCCA (IEEE 802.11e) and without HCCA (IEEE 802.11) for MANET with infrastructure network. The network simulator ns-2 (version 2.29) is used for simulation.

The simulation scenario-1 shown in Figure 1 is for hybrid network. It consists of 18 static nodes in ad hoc wireless domain. Simulation scenario-2 shown in Figure 2, which is different from scenario-1 in the context that

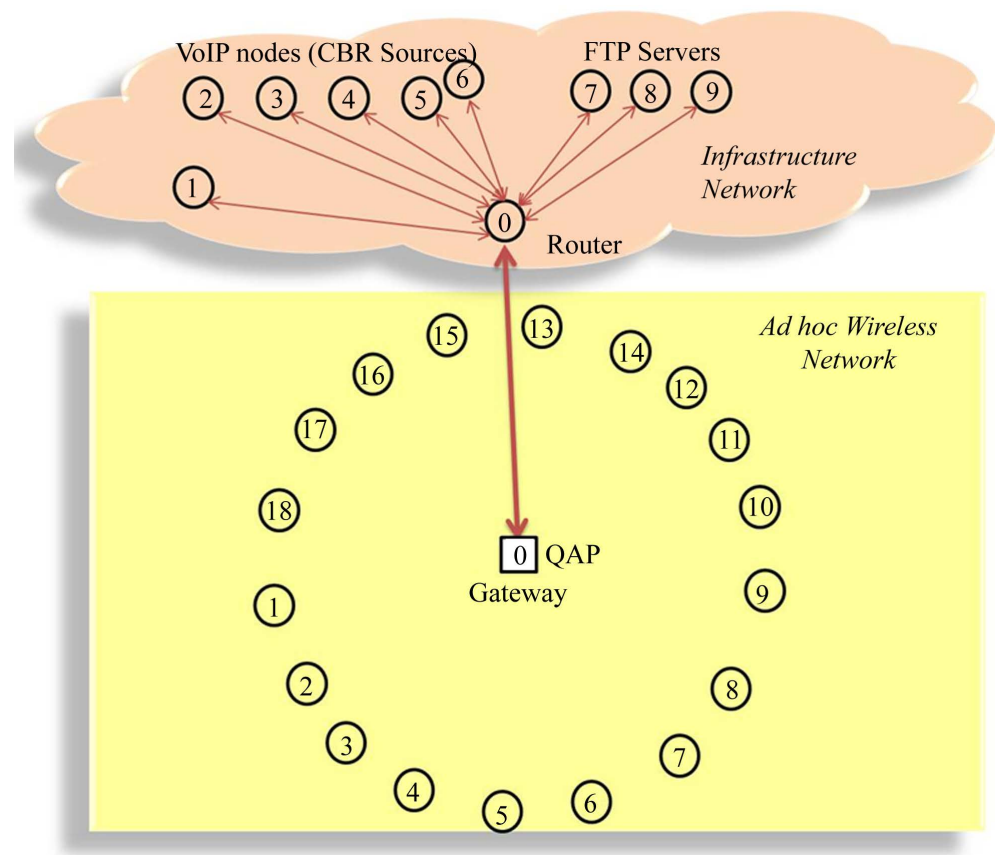

Figure 1. Simulation Scenario-1. 


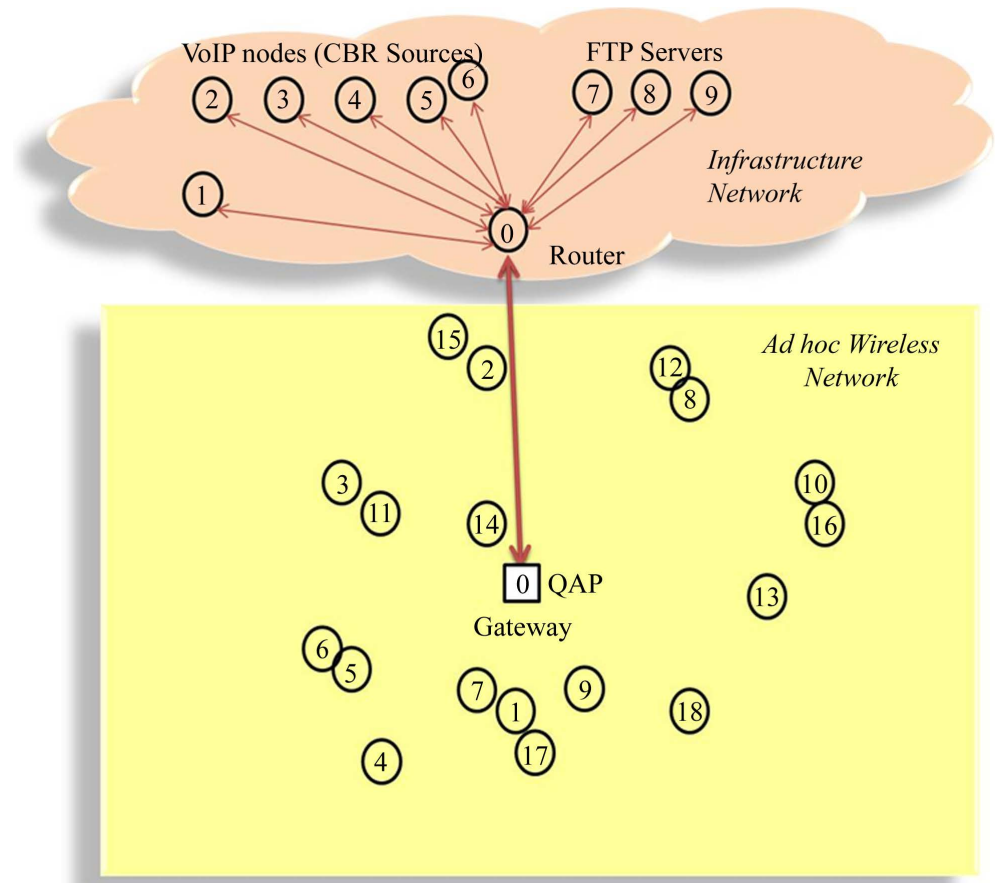

Figure 2. Simulation Scenario-2.

it consist of 18 mobile nodes in ad hoc wireless domain. The rest of the simulation parameters for both the scenarios are same and given in Table 3 . The simulation scenario- $1 \& 2$ contains a gateway, 3 FTP servers and 1 7 real time CBR Sources in infrastructure wired domain. The topology area is $1000 \mathrm{~m} \times 1000 \mathrm{~m}$ is taken for both simulation scenarios. All ad hoc wireless domain nodes can communicate directly with gateway and its direct transmission range is 250 meters. The simulation ran for 100 seconds and the first 20 seconds are considered as warm up time. To achieve this objective, some tests conclude that the first 20 seconds are the transient state of the network during which the connections are set up, so that the first 20 seconds are ignored in simulations. The interest is to study the steady state behavior of the proposed network scenario1 presented in Figure 1.

In order to evaluate the effectiveness of HCCA, these scenarios use real time CBR traffic. The real time CBR traffic is modeled according to VoIP stream based on G.711 voice codec generating 160 bytes every 20 ms, resulting in $64 \mathrm{kbps}$ data rate. This kind of traffic is given higher priority over the other traffic used in the proposed scenario. The another selected application is FTP, which represents a bulk data transfer of large size, sending TCP segments equal to 1024 bytes. FTP is given low priority and this application has always something to send and runs throughout the whole simulation. When CBR sources begin sending data, the QAP gives priority to CBR transmission over FTP data transmission. At that time FTP has to stop its transmission and give the priority to CBR traffic for transmission.

The simulation scenarios presented in Figure 1 and Figure 2 contain 1 to 6 nodes for VoIP communication and remaining three servers (or nodes) involved for FTP in the infrastructure wired domain. The purpose of selecting the varying number of real time VoIP calls was made to demonstrate and analyzed the ability of IEEE 802.11e HCCA.

The VoIP messages are encapsulated in UDP/IP packets while the FTP messages are encapsulated in TCP/IP packets. At the network layer, the extended AODV is used as ad hoc routing protocol with reactive gateway discovery to access the fixed network or vice versa through gateway. At the MAC and physical layer IEEE 802.11e HCCA or IEEE 802.11 without HCCA are used for the evaluation and comparison purpose. The simulation scenarios considered in this paper are influenced by the real time application, such as: where users sit in a cafe, university campus, conference hall, airport, or railway station and access the Internet using their laptops.

\subsection{Performance Metrics}

The following metrics are used in simulation scenarios shown in Figure 1 and Figure 2 for purpose of evalua- 
Table 3. Simulation parameters for Scenario-1 and 2.

\begin{tabular}{cc}
\hline Parameter & Value \\
\hline Topology area & $1000 \mathrm{~m} \times 1000 \mathrm{~m}$ \\
Nodes in ad hoc wireless domain & 18 \\
Gateway & 1 \\
Number of CBR sources & $1-6$ variable \\
Number of FTP sources & 3 \\
CBR packet size and rate & 160 bytes, $64 \mathrm{kbps}$ \\
FTP packet size & 1024 bytes \\
Transmission range & $250 \mathrm{~m}$ \\
Simulation Time & $100 \mathrm{sec}$. \\
Warm up time & $20 \mathrm{sec}$ \\
Speed of nodes (For Scenario 2) & $2 \mathrm{~m} / \mathrm{s}$ \\
\hline
\end{tabular}

tion and performance comparison of extended AODV with HCCA and without HCCA in hybrid network.

Packet Delivery Ratio (PDR) is calculated as the number of data packets received at the destination divided by the number of data packets generated at the source.

End -to- End Delay (EED) is calculated as the time when a packet is received at the destination minus the time when the packet was generated at the source.

Jitter is calculated as the variance of the end-to-end delay.

Throughput is calculated as the number of data bits received at the destination divided by the time the considered traffic type (VoIP, FTP).

\subsection{Simulation Results}

This section presents the simulation results for both the scenarios of the hybrid network. The performance of extended AODV is analyzed and compared with and without HCCA.

\subsubsection{End to End Delay}

The plots of end-to-end delay for scenario 1 (nodes are static in ad hoc wireless domain) are shown in Figure 3 and Figure 4 and for the scenario 2 (nodes are mobile in ad hoc wireless domain) are presented in Figure 5 and Figure 6. The end- to- end delay metric is computed for extended AODV with IEEE 802.11 and 802.11e HCCA MAC. The result of simulation scenario 1 in Figure 3 shows that the end- to- end delay metric values are varying in between 0.02 to 0.12 seconds. This large variation is obtained from extended AODV with IEEE 802.11 MAC, which is unsuitable for real time application. On the other hand, Figure 4 shows that the end- to- end delay is almost constant (0.06 seconds) for extended AODV with IEEE 802.11e HCCA MAC. The end- to- end delay simulation results for scenario 2 are visualized in Figure 5 and Figure 6 are the same as scenario 1. This is because of the reservation to access the medium in IEEE 802.11e (HCCA) MAC for transmission of traffic stream (TS), based on QoS requirements. The Service Interval (SI) value and Transmission Opportunity (TXOP) are predefined by Quality of service Access Point (QAP) for the real time periodic traffic and contention free medium access for CBR traffic. Therefore, allocation of medium periodically to a specific traffic stream by QAP provides almost constant end- to- end delay as desired. An interesting observation that needs to be focused is the sharp increase of end- to- end delay as number of CBR sources increases. This is because that few stations are outside the transmission range of both transmitters and receivers. But with increase in CBR sources, end- to- end delay is very less in the case of IEEE 802.11e (with HCCA) comparing to IEEE 802.11 (without HCCA). The results are comparable for both the scenarios; i.e. whether the nodes in ad hoc wireless network are stationary with specific pattern (Figure 1) or moving in random manner (Figure 2). 


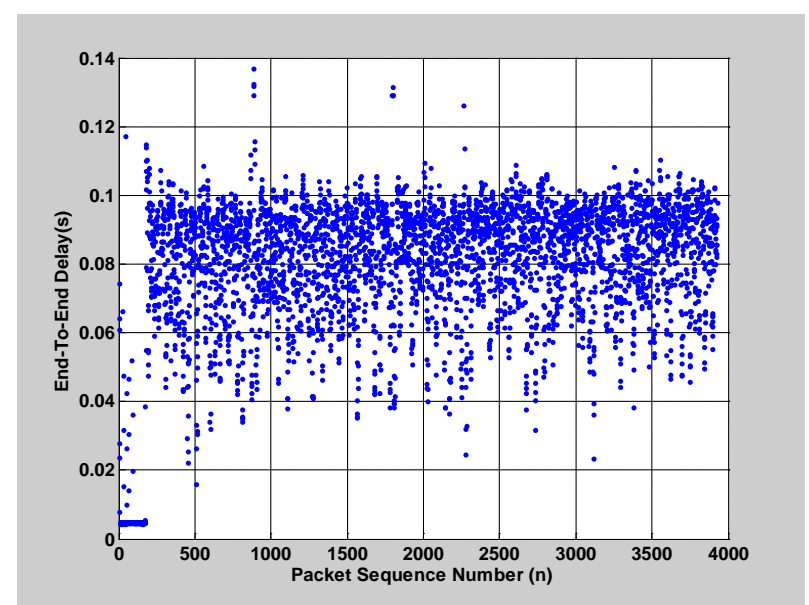

Figure 3. EED plot without HCCA (Scenario-1).

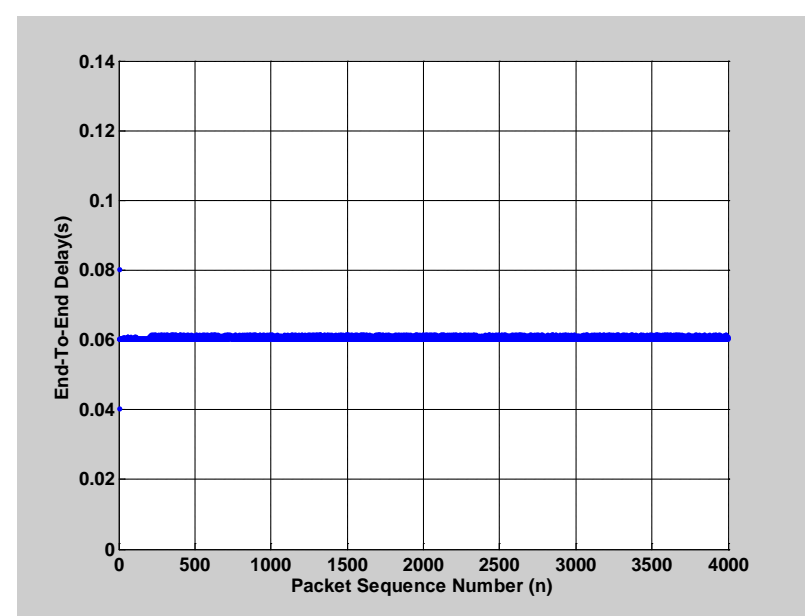

Figure 4. EED plot with HCCA (Scenario-1).

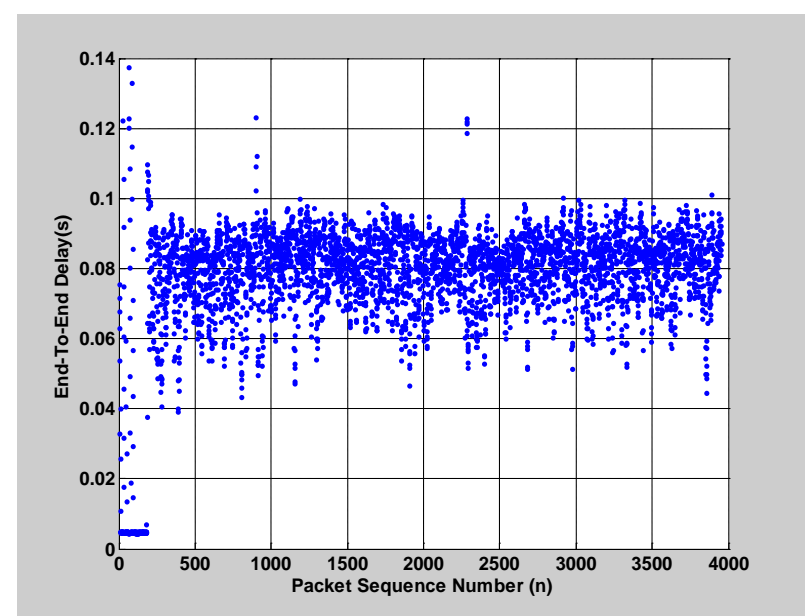

Figure 5. EED plot without HCCA (Scenario-2).

\subsubsection{Jitter}

Jitter results for simulation scenario1 (when nodes are static in ad wireless domain) are given in Table 4(a) and for simulation scenario 2 (when nodes are mobile in ad wireless domain) in Table 4(b). The varying number of 


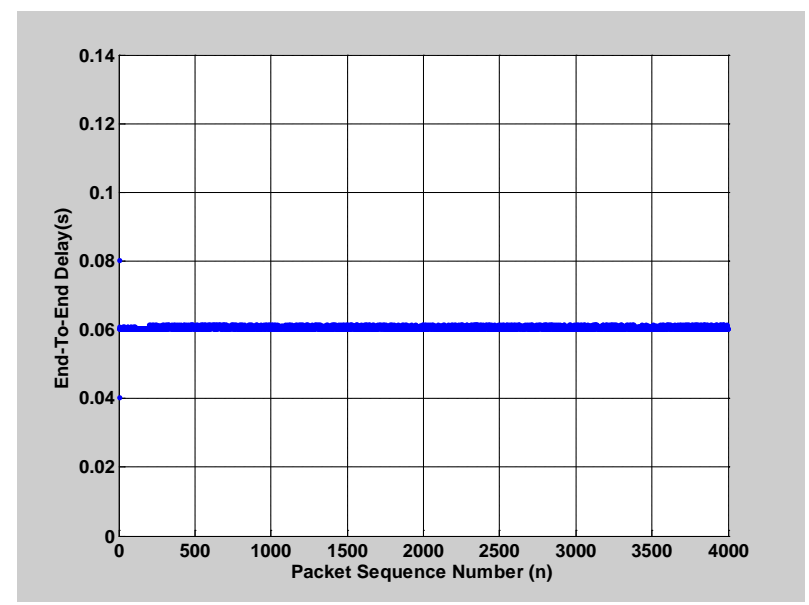

Figure 6. EED plot with HCCA (Scenario-2).

Table 4. (a) Jitter (Scenario-1); (b) Jitter (Scenario-2).

(a)

\begin{tabular}{ccc}
\hline \multirow{2}{*}{$\begin{array}{c}\text { No. of CBR } \\
\text { Sources }\end{array}$} & \multicolumn{2}{c}{ Jitter $\left(10^{-4} \mathrm{~s}^{2}\right)$ for CBR sources } \\
\cline { 2 - 3 } 1 & Without HCCA & With HCCA \\
\hline & 2.7999 & 0.0016 \\
4 & 4.9612 & 0.0035 \\
& 4.9688 & 0.0035 \\
& 5.0256 & 0.0035 \\
& 5.0707 & 0.0015 \\
& 3.1377 & 0.0034 \\
6 & 3.1872 & 0.0036 \\
& 3.1851 & 0.0035 \\
& 3.2014 & 0.0036 \\
& 3.1919 & 0.0033 \\
& 3.1434 & 0.0015 \\
\hline
\end{tabular}

(b)

\begin{tabular}{ccc}
\hline \multirow{2}{*}{$\begin{array}{c}\text { No. of CBR } \\
\text { Sources }\end{array}$} & \multicolumn{2}{c}{ Jitter $\left(10^{-4} \mathrm{~s}^{2}\right)$ for CBR sources } \\
\cline { 2 - 3 } 1 & Without HCCA & With HCCA \\
\hline & 6.69243 & 0.00155 \\
4 & 4.06401 & 0.00351 \\
& 4.11655 & 0.00359 \\
& 4.21340 & 0.00164 \\
& 4.40979 & 0.00161 \\
& 3.02080 & 0.00355 \\
& 3.04994 & 0.00356 \\
6 & 3.11385 & 0.00366 \\
& 3.27290 & 0.08584 \\
& 3.41820 & 0.00165 \\
& 3.69451 & 0.00359 \\
\hline
\end{tabular}

CBR sources from 1 to 6 are taken for simulation of jitter parameter. The computation of Jitter parameter is used to compare the performance of extended AODV with IEEE 802.11 (i.e. Without HCCA) and 802.11e HCCA MAC. The simulation results for the scenarios 1 and 2 implies that as the number of CBR sources are increasing from 1 to 6 , the jitter values are lie in the range of $\left(0.0016 \times 10^{-4} \mathrm{~s}^{2}\right.$ to $\left.0.0034 \times 10^{-4} \mathrm{~s}^{2}\right)$ and $\left(0.00155 \times 10^{-4} \mathrm{~s}^{2}\right.$ to $0.00359 \times 10^{-2} \mathrm{~s}^{2}$ ) with IEEE 802.11e HCCA MAC respectively. On the other hand, with the increasing CBR sources the jitter values are ranging from $\left(2.799 \times 10^{-4} \mathrm{~s}^{2}\right.$ to $\left.5.0707 \times 10^{-4} \mathrm{~s}^{2}\right)$ and $\left(3.69451 \times 10^{-4} \mathrm{~s}^{2}\right.$ to 6.69243 $\times 10^{-4} \mathrm{~s}^{2}$ ) with IEEE 802.11 MAC for both scenarios, shown in Table 4(a) and Table 4(b) respectively. It has 
been examined that as the CBR sources are increasing, the jitter is quite low and almost constant with IEEE 802.11e HCCA MAC compare to the IEEE 802.11 MAC. This proves that the IEEE 802.11e HCCA is able to provide QoS to high priority traffic even during high traffic load in both the scenarios. In contrast to this, the nodes are either mobile or static in ad hoc wireless domain; jitter variation pattern is random and quite high with the IEEE 802.11 and does not provide QoS to the hybrid network. Since the jitter is related with end to end delay, therefore, the similar reason is here to get low jitter value with IEEE 802.11e HCCA MAC and hence is able to provide the QoS to the considered hybrid network scenarios. Therefore, the desirable results are unaffected by the nodes pattern (either static or dynamic) in ad hoc wireless domain in hybrid network.

\subsubsection{Packet Delivery Ratio}

The simulation results obtained for packet delivery ratio versus varying CBR traffic sources are compiled in Table 5(a) and Table 5(b) for simulation scenarios 1 and 2 respectively. As number of CBR sources are varying from 1 to 6, packet delivery ratio goes down (from $96.57 \%$ to $86.35 \%$ ) and (from $96.42 \%$ to $83.59 \%$ ) for scenario 1 and 2 respectively. On the other hand, the packet loss is negligible with IEEE 802.11e MAC (with HCCA) for CBR. It is clear from both tables that as the number of CBR sources are goes on increasing, but the values of packet delivery ratio are constant and near to 99.9\% with IEEE 802.11e MAC (with HCCA) for CBR.

Table 5(a) and Table 5(b) show that PDR results for FTP source is almost same with IEEE 802.11 MAC (i.e. without HCCA) and IEEE 802.11e MAC (i.e. with HCCA). From both the simulation scenarios, PDR values are remain high and unaffected by the varying number of CBR sources. This is due to the reliable delivery service provided by TCP. FTP use connection-oriented TCP, which retransmits dropped packets.

Therefore, it is proved from the simulation results that IEEE 802.11e MAC (with HCCA) providing QoS support to MANET with infrastructure network, either the nodes are mobile or immobile in ad hoc wireless domain.

\subsubsection{Throughput}

Table 6(a) and Table 6(b) are for throughput result analysis of the considered simulation scenario 1 and scenario 2 respectively. It is found that the throughput is slightly better with IEEE 802.11e HCCA compare to the IEEE 802.11 (without HCCA). Since the more number of traffic streams are contending to access the medium which implies the higher probability of collisions and retransmissions resulting in lower throughput for the case of without HCCA. Although, as the traffic streams increases, the throughput is almost consistent and very close to the actual value in case of IEEE 802.11e HCCA. It is visualized from both tables of throughput that IEEE 802.11 HCCA is able to provide required QoS in both the scenarios and has no effect of mobility and non-mobility of nodes in ad hoc wireless domain.

Table 5. (a) Packet Delivery Ratio (Scenario-1); (b) Packet Delivery Ratio (Scenario-2).

(a)

\begin{tabular}{ccccc}
\hline \multirow{2}{*}{$\begin{array}{c}\text { No. of CBR } \\
\text { Sources }\end{array}$} & \multicolumn{2}{c}{ PDR for CBR Sources $(\%)$} & \multicolumn{2}{c}{ PDR for FTP Sources (\%) } \\
\cline { 2 - 5 } & Without HCCA & With HCCA & Without HCCA & With HCCA \\
\hline 1 & 96.57 & 99.93 & 96.53 & 96.96 \\
4 & 91.12 & 99.92 & 95.50 & 96.22 \\
6 & 86.35 & 99.89 & 94.64 & 95.87 \\
\hline
\end{tabular}

(b)

\begin{tabular}{ccccc}
\hline \multirow{2}{*}{$\begin{array}{c}\text { No. of CBR } \\
\text { Sources }\end{array}$} & \multicolumn{2}{c}{ PDR for CBR Sources $(\%)$} & \multicolumn{2}{c}{ PDR for FTP Sources (\%) } \\
\cline { 2 - 5 } & Without HCCA & With HCCA & Without HCCA & With HCCA \\
\hline 1 & 96.42 & 99.93 & 96.49 & 96.92 \\
4 & 88.12 & 99.91 & 94.84 & 96.22 \\
6 & 83.59 & 99.89 & 93.97 & 96.08 \\
\hline
\end{tabular}


Table 6. (a) Throughput (Scenario-1); (b) Throughput (Scenario-2).

(a)

\begin{tabular}{ccccc}
\hline \multirow{2}{*}{$\begin{array}{c}\text { No. of CBR } \\
\text { Sources }\end{array}$} & \multicolumn{2}{c}{ Throughput for CBR Sources (kbps) } & \multicolumn{2}{c}{ Throughput for FTP Sources (kbps) } \\
\cline { 2 - 5 } & Without HCCA & With HCCA & Without HCCA & With HCCA \\
\hline 1 & 61.82 & 63.97 & 3154.94 & 3202.15 \\
4 & 233.33 & 255.86 & 2709.50 & 2910.82 \\
6 & 320.96 & 383.65 & 2313.11 & 2619.70 \\
\hline
\end{tabular}

(b)

\begin{tabular}{ccccc}
\hline \multirow{2}{*}{$\begin{array}{c}\text { No. of CBR } \\
\text { Sources }\end{array}$} & \multicolumn{2}{c}{ Throughput for CBR Sources (kbps) } & \multicolumn{2}{c}{ Throughput for FTP Sources (kbps) } \\
\cline { 2 - 4 } & Without HCCA & With HCCA & Without HCCA & With HCCA \\
\hline 1 & 61.728 & 63.968 & 3251.61 & 3287.04 \\
6 & 225.632 & 255.84 & 2805.25 & 2986.80 \\
\hline
\end{tabular}

\section{Conclusion}

This paper evaluates the ability of extended AODV with IEEE 802.11e HCCA and IEEE 802.11 without HCCA to hybrid networks for two scenarios. The motive is to provide guaranteed quality of service (QoS) support in the considered hybrid network scenarios. This aim is successfully accomplished through extended AODV with IEEE 802.11e HCCA MAC to the hybrid networks. The effectiveness of extended AODV in hybrid networks is evaluated and compared through IEEE 802.11e HCCA MAC and IEEE 802.11 MAC. Simulation results show that the performance is degraded with increasing number of CBR sources when IEEE 802.11 (without HCCA) is used for the hybrid network scenarios, whereas IEEE 802.11e (with HCCA) successfully provides QoS support in terms of low and controlled end-to-end delay and jitter, the required throughput, and negligible packet loss to real time applications. All results obtained from both the scenarios prove that the performance of extended AODV with IEEE 802.11e HCCA improve QoS in hybrid network and it is unaffected whether the nodes are mobile or static in ad hoc wireless domain of hybrid networks.

\section{References}

[1] Hamidian, A. (2003) A Study of Internet Connectivity for Mobile Ad Hoc Networks in NS 2. Master’s Thesis, Lund University, Lund.

[2] Hamidian, A. and Körner, U. (2009) Distributed Reservation-Based QoS in Ad Hoc Networks with Internet Access Connectivity. Proceedings of International Teletraffic Congress, ITC 21, Paris, 15-17 September 2009, 1-5.

[3] Chen, L. and Heinzelman, W.B. (2005) QoS-Aware Routing Based on Bandwidth Estimation for Mobile Ad Hoc Networks. IEEE Journal on Selected Areas in Communications, 23, 561-572.

[4] Hamidian, A. and Körner, U. (2006) An Enhancement to the IEEE 802.11e EDCA Providing QoS Guarantees. Telecommunication Systems, 31, 195-212. http://dx.doi.org/10.1007/s11235-006-6520-Z

[5] Bin Muhamad Noh, Z.A., Suzuki, T. and Tasaka, S. (2006) A Packet Scheduling Scheme for Audio-Video Transmission with IEEE 802. 11e HCCA and Its Application-Level QoS Assessment. Asia-Pacific Conference on Communications, APCC’06, Busan, 31 August-1 September 2006, 1-5.

[6] Grilo, A., Macedo, M. and Nunes, M. (2003) A Scheduling Algorithm for QoS Support in IEEE 802.11e Networks. IEEE Wireless Communications, 10, 36-43. http://dx.doi.org/10.1109/MWC.2003.1209594

[7] Liang, H. and Zeng, F. (2012) A Research on HCCA Mechanism of Wireless LAN Access. Journal of Networks, 7, 845-849.

[8] Singh, S. and Tripathi, R. (2013) Optimization in Route Discovery Delay for Integrated MANET with Internet Using Extended AODV. International Journal of Computer Applications, 65, 16-22.

[9] Ruscelli, A.L., Cecchetti, C., Alifano, A. and Lipari, G. (2012) Enhancement of QoS Support of HCCA Schedulers Using EDCA Function in IEEE 802.11e Networks. Ad Hoc Networks, 10, 147-161. 
http://dx.doi.org/10.1016/j.adhoc.2010.09.014

[10] Hsieh, J.-R. and Lee, T.-H. (2007) Data Rate Estimation Algorithm for IEEE 802.11e HCCA Scheduler. International Journal of Pervasive Computing and Communications, 3, 243-256. http://dx.doi.org/10.1108/17427370710856219

[11] Hamidian, A. and Körner, U. (2008) Extending EDCA with Distributed Resource Reservation for QoS Guarantees. Telecommunication Systems, 39, 87-194. http://dx.doi.org/10.1007/s11235-008-9124-y

[12] Ni, Q., Romdhani, L. and Turletti, T. (2004) A Survey of QoS Enhancements for IEEE 802.11 Wireless LAN. Journal of Wireless Communications and Mobile Computing, 4, 547-566.

[13] Ni, Q. (2005) Performance Analysis and Enhancements for IEEE 802.11e Wireless Networks. IEEE Network, 19, 2127. http://dx.doi.org/10.1109/MNET.2005.1470679

[14] Skyrianoglou, D., Passas, N. and Salkintzis, A.K. (2006) ARROW: An Efficient Traffic Scheduling Algorithm for IEEE 802.11e HCCA. IEEE Transaction on Wireless Communications, 5, 3558-3567. http://dx.doi.org/10.1109/TWC.2006.256978

[15] Jansang, A. and Phonphoem, A. (2011) Adjustable TXOP Mechanism for Supporting Video Transmission in IEEE 802.11e HCCA. EURASIP Journal on Wireless Communications and Networking, 2011, 158. 